\title{
Phase relationships of spectral oscillations in 2D molecular spectroscopy
}

\author{
Vytautas Butkus $^{1,2, a}$, Donatas Zigmantas ${ }^{3}$, Leonas Valkunas ${ }^{1,2}$, and Darius Abramavicius ${ }^{1,4}$ \\ 1 Faculty of Physics, Vilnius University, Lithuania \\ 2 Center for Physical Sciences and Technology, Vilnius, Lithuania \\ 3 Department of Chemical Physics, Lund University, Sweden \\ 4 Key Laboratory of Supramolecular Complexes, Jilin University, Changchun, PR China
}

\begin{abstract}
Spectral oscillations in 2D molecular spectroscopy of the displaced oscillator system and their phase relationships are analyzed. The phase maps of the dispersive and absolute-valued signals give hints for experimental probing of peak oscillations.
\end{abstract}

\section{Introduction}

Two-dimensional (2D) electronic spectroscopy is a powerful tool capable of resolving ultrafast phenomena such as quantum correlations, energy transfer and decoherence in molecular aggregates [1, 2]. The coherent state evolution appears as slowly-decaying spectral beats in a number of molecular systems. As it was recently shown both experimentally and theoretically the high-frequency molecular vibrations are crucial in shaping the oscillatory behavior of peaks in the 2D spectrum [3]. However, the problem of assignment of the beats to either electronic coherences or molecular vibrations is still debated. Moreover, it is disputed whether such detachment is feasible at all.

In various studies, beats in the dispersive or amplitude signals are analyzed. They correspond to the real or absolute value of the complex 2D signal, respectively. The rephasing, nonrephasing and total signals are also considered. Here the analysis of the spectral oscillation phases is presented; from the oscillation phase maps of the displaced oscillator system the typical phase relationship trends are shown for the real, imaginary and absolute-valued spectra.

\section{Oscillations in the 2D spectra of the displaced oscillator model system}

If we neglect environment-induced relaxation, the rephasing signal within the second-order response function approach is given as the sums of resonant contributions. One such contribution

$$
S\left(\omega_{3}, t_{2}, \omega_{1}\right)=\iint \mathrm{d} t_{1} \mathrm{~d} t_{3} \mathrm{e}^{+\mathrm{i} \omega_{3} t_{3}+\mathrm{i} \omega_{1} t_{1}} \times\left[G_{3}\left(t_{3}\right) G_{2}\left(t_{2}\right) G_{1}\left(t_{1}\right)\right]
$$

is responsible for a single oscillating cross-peak in the 2D spectrum at $\left(\omega_{1}, \omega_{3}\right)=\left(\varepsilon_{1}, \varepsilon_{3}\right)$, where $\varepsilon_{j}$ determines the exciton excitation energies. The cross-peaks in the 2D spectra are usually considered as a direct reflection of the excitation coherence in molecular aggregates. Eq. (1) can be analytically integrated for the density operator propagator chosen as $G_{j}\left(t_{j}\right) \propto \exp \left(-\mathrm{i} \varepsilon_{j} t_{j}-\gamma_{j} t_{j}\right)$ and $\gamma \equiv \gamma_{1}=\gamma_{3}$. Shifting the origin of $\left(\omega_{1}, \omega_{3}\right)$ plot to the peak center is done here by introducing the displacements $\left(\omega_{1}+\varepsilon_{1} \equiv \mp s_{1}\right.$ and $\omega_{3}-\varepsilon_{3} \equiv s_{3}$. Thus, the real and imaginary parts of the cross-peak can be given by

$$
S_{\mathrm{Re} \mid \mathrm{Im}}\left(s_{3}, t_{2}, s_{1}\right)=L_{\mathrm{Re} \mid \mathrm{Im}}\left(s_{1}, s_{3}\right) \mathrm{e}^{-\gamma_{2} t_{2}} \cos \left[\left|\varepsilon_{2}\right| t_{2}+\phi_{\mathrm{Re} \mid \mathrm{Im}}\left(s_{1}, s_{3}\right)\right],
$$

a e-mail: vytautas.butkus@ff.vu.1t

This is an Open Access article distributed under the terms of the Creative Commons Attribution License 2.0, which permits unrestricted use, distribution, and reproduction in any medium, provided the original work is properly cited. 

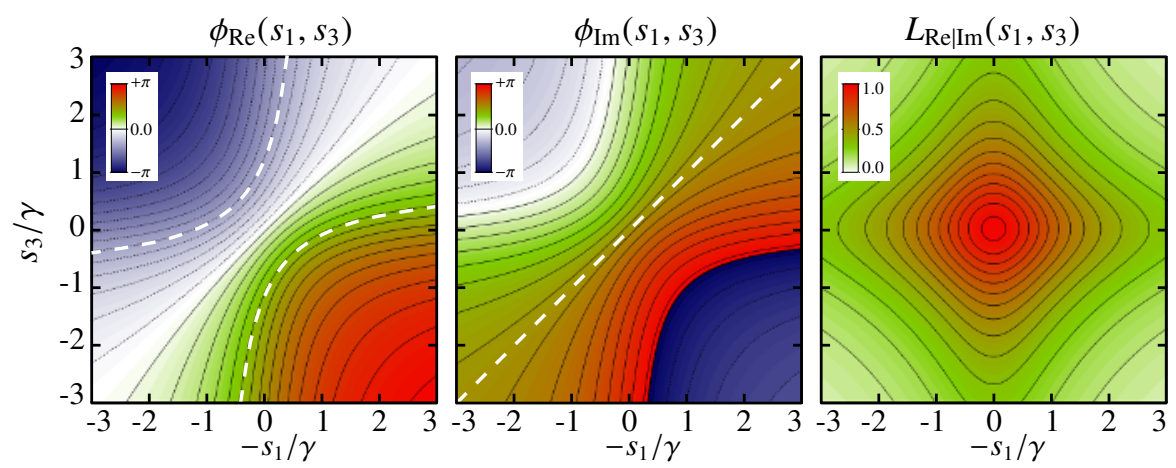

Fig. 1. Amplitude and phase maps of a single cross-peak oscillations in the rephasing signal. White dashed contours correspond to $\pm \frac{\pi}{2}$ phase.

where the lineshape and the phase are defined accordingly

$$
\begin{gathered}
L_{\mathrm{Re}}\left(s_{1}, s_{3}\right)=L_{\operatorname{Im}}\left(s_{1}, s_{3}\right)=\frac{\left[\left(\gamma^{2}-s_{1} s_{3}\right)^{2}+\gamma^{2}\left(s_{3}+s_{1}\right)^{2}\right]^{\frac{1}{2}}}{\left(s_{3}^{2}+\gamma^{2}\right)\left(s_{1}^{2}+\gamma^{2}\right)}, \\
\phi_{\operatorname{Re}}\left(s_{1}, s_{3}\right)=-\operatorname{sgn}\left(s_{3}+s_{1}\right) \cdot \arccos \left(\frac{\gamma^{2}-s_{1} s_{3}}{\left[\left(\gamma^{2}-s_{1} s_{3}\right)^{2}+\gamma^{2}\left(s_{3}+s_{1}\right)^{2}\right]^{\frac{1}{2}}}\right), \\
\phi_{\operatorname{Im}}\left(s_{1}, s_{3}\right)=-\operatorname{sgn}\left(s_{1} s_{3}-\gamma^{2}\right) \cdot \arccos \left(\frac{s_{3} \gamma+s_{1} \gamma}{\left[\left(\gamma^{2}-s_{1} s_{3}\right)^{2}+\gamma^{2}\left(s_{3}+s_{1}\right)^{2}\right]^{\frac{1}{2}}}\right) .
\end{gathered}
$$

The $L_{\mathrm{Re} \mid \mathrm{Im}}\left(s_{1}, s_{3}\right)$ expression is a positive function, while the values of $\phi_{\operatorname{Re} \mid \operatorname{Im}}\left(s_{1}, s_{3}\right)$ vary in the range of $(-\pi \ldots \pi)$. Since they perceive the amplitude and phase of spectral oscillations in 2D spectra, the corresponding scaled amplitude and phase maps can be plotted as shown in Fig. 1. It immediately follows, that the observed phase of the oscillating cross-peak is sensitive to the displacement from the spectral resonance as it is highlighted in Ref. [4].

Here we consider the displaced oscillator model system, which corresponds to a single molecular chromophore strongly bound to a single high-frequency vibronic mode. The $2 \mathrm{D}$ spectra of such a system is obtained by assuming the realistic bath-induced broadening description within the frame of the second-order cumulant expansion technique, which is exact for a monomer system [5]. It is known that the time-resolved 2D spectrum of such system demonstrates oscillations of all peaks and the phases of the spectral beats can be either equal or shifted by $\pi$ [4]. We consider the phase and amplitude maps of the oscillations in spectra of the displaced oscillator. The Fourier tranform of every point in the real, imaginary and absolute value of the $2 \mathrm{D}$ spectrum and the phase and amplitude are reconstructed from the complex value at frequency $\omega_{0}$. In Fig. 2 they are denoted by $\phi_{\mathrm{Re}}^{\mathcal{F}}, \phi_{\mathrm{Im}}^{\mathcal{F}}$, and $\phi_{\mathrm{Abs}}^{\mathcal{F}}$, respectively. The corresponding amplitude maps are identified as $L_{\mathrm{Re}}^{\mathcal{F}}, L_{\mathrm{Im}}^{\mathcal{F}}$, and $L_{\mathrm{Abs}}^{\mathcal{F}}$.

The maps of real and imaginary parts in Fig. 2 clearly show the typical phase and amplitude dependency trends of the cross-peaks as indicated in Fig. 1. In the real part of the rephasing signal, the lower diagonal peak as well as the cross-peak at $\left(-\omega_{1}+\omega_{\text {eg }}, \omega_{3}-\omega_{\text {eg }}\right)=\left(\omega_{0},-\omega_{0}\right)$ oscillates as cosine with the $\pi$ phase shift, while the oscillations of all other peaks are of the zero phase shift. The similar trend continues in the absolute-valued spectrum, however, the phases are slightly more distorted there and the $\pi$ and 0 phase resonances are not situated at the very centers of the peaks. Moreover, the phases in the absolute-valued spectrum can be reported as very sensitive to the system and laser parameters (not shown here). 

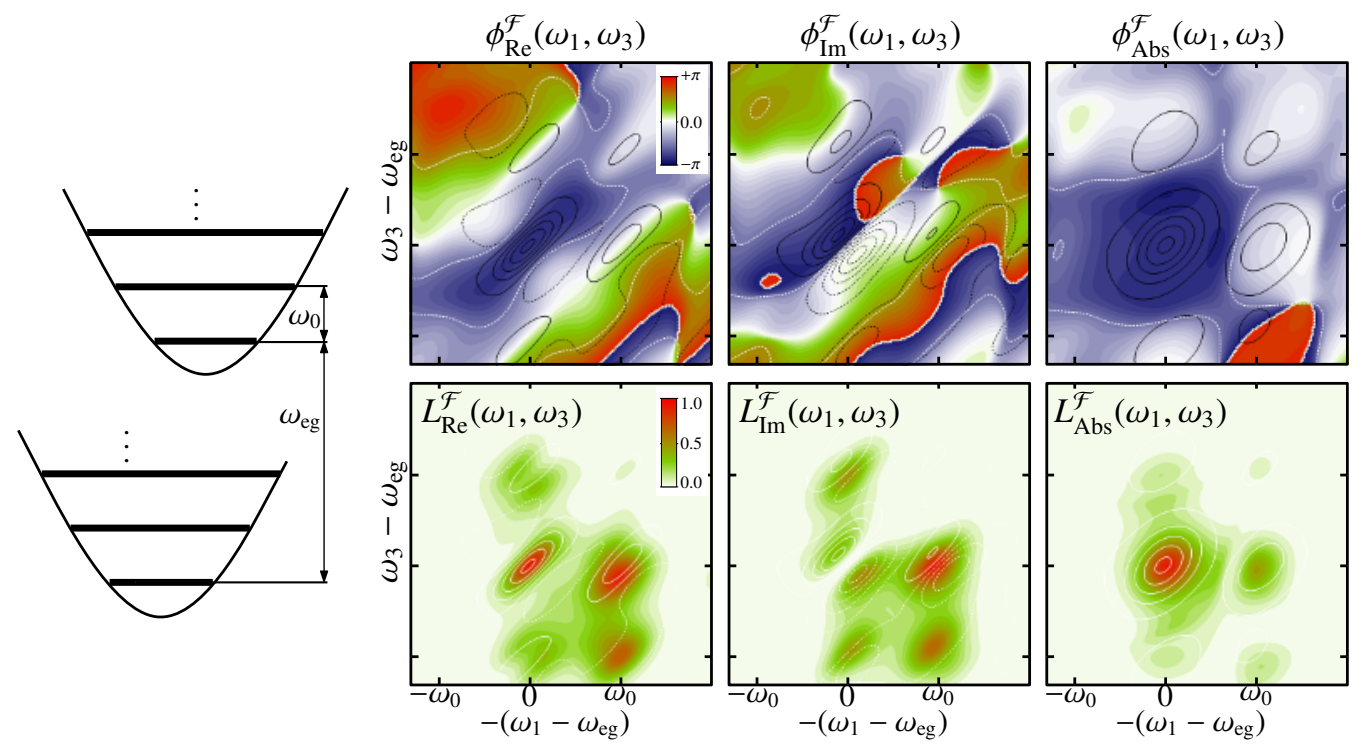

Fig. 2. Amplitude and phase maps of the rephasing 2D spectrum of the displaced oscillator. 2D spectra at $t_{2}=0$ are shown as black and white contours in the phase and amplitude maps, respectively. Dashed white contours indicate the $\pm \frac{\pi}{2}$ phase.

\section{Conclusions}

The phase dependencies of the cross-peak oscillations should be carefully considered when probing the oscillatory dynamics of spectral beats in the 2D molecular spectroscopy. These cross-peak features are very important when the analysis of oscillations are carried out on purpose to distinguish between the coherent dynamics of the electronic and vibronic origins of the system under consideration. If the absolute signal of the $2 \mathrm{D}$ spectrum is considered, these phase map properties become crucial since the real and imaginary parts of the spectroscopic signal are related in a non-trivial way, thus, making the phase map very sensitive to various system and laser pulse parameters.

This research was supported by the European Social Grant under the Global Grant Measure.

\section{References}

1. O. Bixner, V. Lukeš, T. Mančal, J. Hauer, F. Milota, M. Fischer, I. Pugliesi, M. Bradler, W. Schmid, E. Riedle, H. F. Kauffmann, N. Christensson, J. Chem. Phys. 136, 204503 (2012)

2. J. Dostál, T. Mančal, R. Augulis, F. Vácha, J. Pšenčík, D. Zigmantas, J. Am. Chem. Soc. (2012) in press

3. T. Mančal, N. Christensson, V. Lukeš, F. Milota, O. Bixner, H. F. Kauffmann, J. Hauer, J. Phys. Chem. Lett. 3, 1497 (2012)

4. V. Butkus, D. Zigmantas, L. Valkunas, D. Abramavicius, Chem. Phys. Lett. (2012) doi:10.1016/j.cplett.2012.07.014

5. V. Butkus, L. Valkunas, D. Abramavicius, J. Chem. Phys. (2012) in press 